\title{
The effect of isoscalar-isovector coupling in infinite nuclear matter
}

\author{
S. K. Singh ${ }^{1}$, M. Bhuyan ${ }^{1,2}$, P. K. Panda ${ }^{3}$, and S. K. Patra ${ }^{1}$ \\ 1 Institute of Physics, Sachivalaya Marg, Bhubaneswar-751 005, India. \\ ${ }^{2}$ School of Physics, Sambalpur University, Jyotivihar, Burla-768 019, India. \\ ${ }^{3}$ Department of Physics, C. V. Raman College of Engineering, Bhubaneswar-752054, India.
}

(Dated: July 6, 2018)

\begin{abstract}
Working on the framework of Relativistic Mean Field theory, we exposed the effect of nonlinear isoscalarisovector coupling on $\mathrm{G} 2$ parameter set on the density dependence of nuclear symmetry energy in infinite nuclear matter. The observables like symmetric energy and few related coefficients are studied systematically. We presented the results of stiff symmetry energy at sub-saturation densities and a soft variation at normal densities. Correlation between the symmetric energy and the isoscalar-isovector coupling parameter fully demonstrated for wide range of density. The work further extended to the octet system and showed the effect of coupling over the equation of state.
\end{abstract}

PACS numbers: 21.65.Ef, 21.10.Gv, 21.65.+f, 26.60.+c

\section{INTRODUCTION}

The best possible and well defined theoretical laboratory to studied for many body system is infinite nuclear matter at certain conditions. To review the status of microscopic studies of nuclear matter and neutron rich matter, that already reaches to the destination by mean-field models (relativistic and non-relativistic) [1-6] and although by some othermicroscopic, methods such as Brueckner-Hartree-Fock (BHF) [7-10] and Dirac-Brueckner-Hartree-Fock (DBHF) [11-15]. The isospin and density dependence of the nuclear symmetry energy, $E_{\text {sym }}$, is the current interest because of its implications not only in nuclear physics but also in astrophysics. The softening in equation of state (EOS) of nuclear matter likely lead to an exciting problem in astro-nuclear physics from few decades to till date. Mean while the novel phenomena like formation of superheavy nuclei in astrophysical system is the front learning about the island of stability for that regions.

The prediction of compressional modulus $\left(K_{0}\right)$ in symmetric nuclear matter for nonrelativistic and relativistic models differ by about 25 in magnitude. In other word, the nonrelativistic models predict $K_{0} \sim 220-235 \mathrm{MeV}$ [4-6], relativistic models argue for $K_{0} \sim 250-280 \mathrm{MeV}$ [1-3]. The density dependence of the symmetry energy $E_{\text {sym }}$ is not exposed fully at present, which may be responsible for the above discrepancy [16]. Note that the $E_{\text {sym }}$ is the difference between the energy of pure neutron matter and normal nuclear matter, which is a tremendous approximation [3, 17, 18]. The large value of $K_{0}$ shows appropriately stiff symmetric energy i.e. rises rapidly with baryon density [16, 17]. This make a passing reference between $K_{0}$ and $E_{\text {sym }}$, which is a function of density and isospin component of scalar and vector mesons. Thus, it is enlightening to explore the effects of $E_{\text {sym }}$ in the RMF model, which was limited to a narrow range from the analysis of skin data, on the composition and structure of hot proton neutron and cold neutron star matter that hold a large density range [19-23].

In this work, we introduce an extra term on the top of the $G 1$ or $G 2$ forces to the Lagrangian, which is the combined effect of scalar and vector field with isoscalar- isovector coupling constant $\left(\Lambda_{v}\right)$. The newly added term affect to the sym- metric energy and the slope parameter $L_{\text {sym }}$. The problem is the stiffness of the $E_{\text {sym }}$ with respect to baryonic density followed a softer path with respect to density along with this extra added coupling. Without this additional coupling constants one may not be overcome the hindrance in the nuclear matter. The most important prospectus of the coupling constant is that the various other properties and nuclear matter observables are not seriously compromising in their magnitude.

\section{THE RELATIVISTIC MEAN FIELD (RMF) THEORY}

The complete description of EOS can be implemented by using quantum chromodyanmics (QCD), which is the fundamental theory of the strong interaction. The concept of effective field theory (EFT) is applicable to low energy QCD [24] and is called as quantum hadrodynamics (QHD) [25-29]. The description of normal nuclear matter properties [25, 30] and finite nuclei [31-34] are successfully explained by QHD. The exchange of mesons like iso-scalar scalar $\sigma$, iso-scalar vector $\omega$, iso-vector vector $\rho$ and the pseudoscalar $\pi$ are responsible for nucleon interaction and increases the degree of freedom. The nucleons are considered as Dirac spinors moving in classical meson fields. In mean-field approximation, the contribution of the $\pi$ meson is zero, because of its pseudospin nature. The extension of the standard relativistic mean-field (RMF) theory with addition of non-linear scalar-vector and vectorvector self interaction is called as E-RMF proposed by Furnstahl, Serot, and Tang [35, 36] (see also Miller and Serot [37]. The coupling constants are self-consistent with the underlying symmetries of QCD by non-renormalization. The concept of naturalness can focus by expanding the non-linear Lagrangian and organized it in increasing power of field and their derivative according to their dimensional analysis [38, 39] and truncated at a given level of accuracy [40-42]. Here we have taken the fourth order of expanded field and in addition to this we have taken the special interaction between the $\omega$ and $\rho$ with a new coupling constant $\Lambda_{v}$ to get good result in the study of $E_{\text {sym }}$ and other co-efficients. The modified Lagrangian is given as [43, 44]: 


$$
\begin{aligned}
\mathcal{L}= & \sum_{B} \bar{\psi}\left(\left\{i \gamma^{\mu} D_{\mu}-m_{B}+g_{\sigma} \sigma\right) \psi\right. \\
& +\frac{1}{2} \partial_{\mu} \sigma \partial_{\mu} \sigma-m_{\sigma}^{2} \sigma^{2}\left(\frac{1}{2}+\frac{\kappa_{3}}{3 !} \frac{g_{\sigma} \sigma}{m_{B}}+\frac{\kappa_{4}}{4 !} \frac{g_{\sigma}^{2} \sigma^{2}}{m_{B}^{2}}\right) \\
& -\frac{1}{4} \Omega_{\mu \nu} \Omega^{\mu \nu}+\frac{1}{2} m_{\omega}^{2} \omega_{\mu} \omega^{\mu}\left(1+\eta_{1} \frac{g_{\sigma} \sigma}{m_{B}}+\frac{\eta_{2}}{2} \frac{g_{\sigma}^{2} \sigma^{2}}{m_{B}^{2}}\right) \\
& -\frac{1}{4} R_{\mu \nu}^{a} R^{\mu \nu a}+\frac{1}{2} m_{\rho}^{2} \rho_{\mu}^{a} \rho^{a \mu}\left(1+\eta_{\rho} \frac{g_{\sigma} \sigma}{m_{B}}\right) \\
& +\frac{1}{4 !} \zeta_{0} g_{\omega}^{2}\left(\omega_{\mu} \omega^{\mu}\right)^{2}+\Lambda_{v}\left(g_{\rho}^{2} \rho_{\mu}^{a} \rho^{\mu a}\right)\left(g_{\omega}^{2} \omega_{\mu} \omega^{\mu}\right)
\end{aligned}
$$

The co-variant derivative $D_{\mu}$ is defined as

$$
D_{\mu}=\partial_{\mu}+i g_{\omega} \omega_{\mu}+i g_{\rho} I_{3} \tau^{a} \rho_{\mu}^{a},
$$

where $R_{\mu \nu}^{a}$ and $\Omega_{\mu \nu}$ are field tensors and defined as follow

$$
\begin{gathered}
R_{\mu \nu}^{a}=\partial_{\mu} \rho_{\nu}^{a}-\partial_{\nu} \rho_{\mu}^{a}+g_{\rho} \epsilon_{a b c} \rho_{\mu}^{b} \rho_{\nu}^{c}, \\
\Omega_{\mu \nu}=\partial_{\mu} \omega_{\nu}-\partial_{\nu} \omega_{\mu},
\end{gathered}
$$

Here $m_{B}$ is the mass of nucleon, and $m_{\sigma}, m_{\omega}$ and $m_{\rho}$ are the masses of corresponding mesons. The equation of motion derived from the Lagrangian using mean-field approximation self-consistently. The field equation for different meson fields are given by

$$
\begin{gathered}
m_{\sigma}^{2}\left(\sigma_{0}+\frac{g_{\sigma} \kappa_{3} \sigma_{0}^{2}}{2 m_{B}^{2}}+\frac{g_{\sigma}^{2} \kappa_{4} \sigma_{0}^{3}}{6 m_{B}^{2}}\right) \\
-\frac{1}{2} m_{\omega}^{2}\left(\eta_{1} \frac{g_{\sigma}}{m_{B}}+\eta_{2} \frac{g_{\sigma}^{2} \sigma_{0}}{m_{B}^{2}}\right) \omega_{0}^{2} \\
-\frac{1}{2} m_{\rho}^{2} \eta_{\rho} \frac{g_{\sigma} \rho_{0}^{2}}{m_{B}}=\sum g_{\sigma} \rho_{s} \\
m_{\omega}^{2}\left(1+\eta_{1} \frac{g_{\sigma} \sigma_{0}}{m_{B}}+\eta_{2} \frac{g_{\sigma}^{2} \sigma_{0}^{2}}{m_{B}^{2}}\right) \omega_{0} \\
-\frac{1}{6} \zeta_{0} g_{\omega}^{2} \omega_{0}^{3}+2 \Lambda_{v} g_{r h o}^{2} \rho_{0}^{2} g_{\omega}^{2} \omega_{0}=\sum g_{\omega} \rho_{B} \\
{\left[m_{\rho}^{2}\left(1+\eta_{\rho} \frac{g_{\sigma} \sigma_{0}}{m_{B}}\right)+2 \Lambda_{v} g_{r h o}^{2} g_{\omega}^{2} \omega_{0}^{2}\right] \rho_{0}=\frac{1}{2} \sum g_{\rho} I_{3} \rho(7)}
\end{gathered}
$$

The scalar $\left(\rho_{s}\right)$ and vector $(\rho)$ densities are given as follow:

$$
\rho_{s}=\frac{1}{\pi^{2}} \int_{0}^{k_{B}} \frac{k^{2} d k}{E_{B}^{*}}
$$

and

$$
\rho_{B}=\frac{1}{\pi^{2}} \int_{0}^{k_{B}} k^{2} d k
$$

where, $\left.E_{B}^{*}=\sqrt{(} k^{2}+m_{B^{* 2}}\right)$ is the effective energy, $k_{B}$ is the Fermi momentum, $m_{B}^{*}=m_{B}-g_{\sigma} \sigma$ stands for effective mass (which solved self-consistently), $I_{3}$ is the third component of isospin projection and $B$ stands for baryon. The energy and pressure density depends on the effective mass $m_{B}^{*}$ of the system, so first need to solve this self-consistent Eqn. (4). As the self-consistent field obtained, the expression for energy and pressure density are given as;

$$
\begin{aligned}
\mathscr{E}= & \sum_{B} \frac{2}{(2 \pi)^{3}} \int_{0}^{k_{B}} d^{3} k E_{B}^{*}(k)+\frac{1}{8} \zeta_{0} g_{\omega}^{2} \omega_{0}^{4} \\
& +m_{\sigma}^{2} \sigma_{0}^{2}\left(\frac{1}{2}+\frac{\kappa_{3}}{3 !} \frac{g_{\sigma} \sigma}{m_{B}}+\frac{\kappa_{4}}{4 !} \frac{g_{\sigma}^{2} \sigma^{2}}{m_{B}^{2}}\right) \\
& +\frac{1}{2} m_{\omega}^{2} \omega_{0}^{2}\left(1+\eta_{1} \frac{g_{\sigma} \sigma}{m_{B}}+\frac{\eta_{2}}{2} \frac{g_{\sigma}^{2} \sigma^{2}}{m_{B}^{2}}\right) \\
& +\frac{1}{2} m_{\rho}^{2} \rho_{0}^{2}\left(1+\eta_{\rho} \frac{g_{\sigma} \sigma}{m_{B}}\right) \\
& +3 \Lambda_{v} \Omega_{0}^{2} R_{0}^{2}+\sum_{l} \varepsilon_{l},
\end{aligned}
$$

and

$$
\begin{aligned}
\mathscr{P}= & \sum_{B} \frac{2}{3(2 \pi)^{3}} \int_{0}^{k_{B}} d^{3} k E_{B}^{*}(k)+\frac{1}{8} \zeta_{0} g_{\omega}^{2} \omega_{0}^{4} \\
& +m_{\sigma}^{2} \sigma_{0}^{2}\left(\frac{1}{2}+\frac{\kappa_{3}}{3 !} \frac{g_{\sigma} \sigma}{m_{B}}+\frac{\kappa_{4}}{4 !} \frac{g_{\sigma}^{2} \sigma^{2}}{m_{B}^{2}}\right) \\
& +\frac{1}{2} m_{\omega}^{2} \omega_{0}^{2}\left(1+\eta_{1} \frac{g_{\sigma} \sigma}{m_{B}}+\frac{\eta_{2}}{2} \frac{g_{\sigma}^{2} \sigma^{2}}{m_{B}^{2}}\right) \\
& +\frac{1}{2} m_{\rho}^{2} \rho_{0}^{2}\left(1+\eta_{\rho} \frac{g_{\sigma} \sigma}{m_{B}}\right) \\
& +\Lambda_{v} \Omega_{0}^{2} R_{0}^{2}+\sum_{l} P_{l},
\end{aligned}
$$

where $P_{l}$ and $\varepsilon_{l}$ are lepton's pressure and energy density, respectively (and here we have taken electron only). Note that the following quantities are redefined as, $\Omega_{0} \equiv g_{\omega} \omega_{0}$ and $R_{0} \equiv g_{\rho} \rho_{03}$. At zero temperature, the pressure of the system obtained from energy density and its first derivative, which is thermodynamically consistent in mean-field (MF) approximation.

\section{CALCULATIONS AND DISCUSSION}

We have solved the mean field equations self-consistently and parametrized the coupling constant by renormalization. We have made attention to $\mathrm{G} 2[35,36]$ parametrization sets of the E-RMF model to reproduce the fundamental properties of nuclear matter. In this parameter set $\zeta$ coupling constant is determined by self-interactions of $\omega$-meson, which is responsible for the softening the EOS at high density and reproduced the maximum mass of neutron star. For softening the symmetric energy of the system at nuclear matter density, 

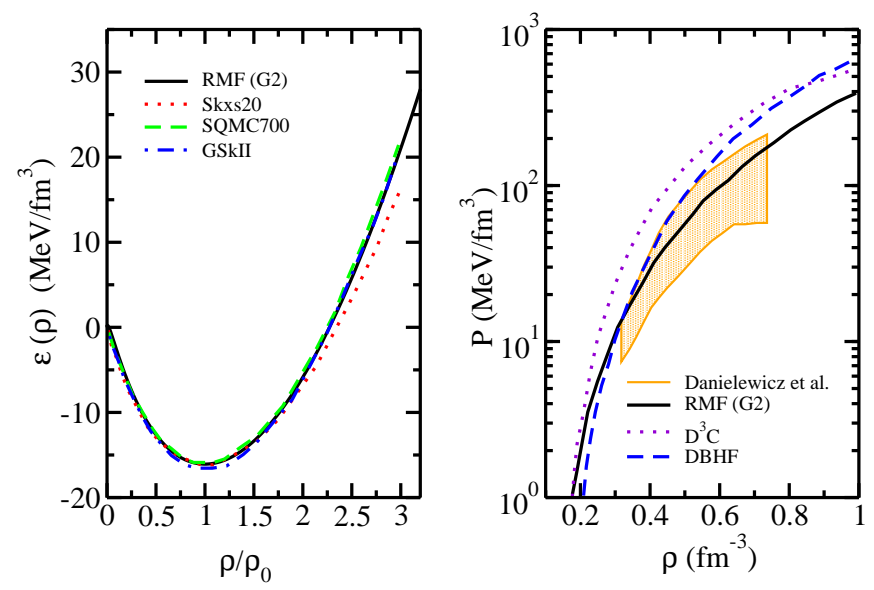

FIG. 1: The EOS or energy per nucleon and pressure density as a function of density from RMF compare with the other predictions.

we have added an extra coupling between iso-scalar and isovector field with coupling constant $\Lambda_{v}$. This made make possible to soften the $E_{\text {sym }}$ and reproduced saturation properties for the force parameter at $\Lambda_{v} \sim 0.00$. In other word, $\Lambda_{v}$ is simply a self interaction constant between isoscalar and isovector field, which does not change any other nuclear matter properties like energy density, pressure density and so many considerably.

\section{A. Energy and Pressure density}

Infinite nuclear matter is important for the investigation of physical quantities relevant to heavy nuclei and compact objects like neutron star. At saturation density $\rho_{0}$, the binding energy per particle $\epsilon$ as a function of density is well established quantities consistent to thermodynamic relation at certain temperature. These translational invariance of the system facilitates theoretical calculations of these observables. The prediction of E-RMF $\left(\mathrm{G} 2+\Lambda_{v}\right)$ for EOS of nuclear matter (NM) (solid black line) compared with DBHF [45] and other theoretical predictions [46] are in Fig. 1 (a). From the figure, it is clear that all the prediction well matches with respect to density. Fig. 1(b), shows the comparison of pressure density with experimental data [47] and theoretical predictions [45, 48, 49]. The obtained results for energy density (see Fig. 1) from Ref. [21] is exactly same to the present calculation. This implies that the EOS of SNM is not affected by the new coupling with $\Lambda_{v}$. Being insensitive to this observable, the aim of this present work to pursue the systematic variation of $E_{\text {sym }}$ by employing the isoscalar-isovector coupling parameter $\Lambda_{v}$, which discussed below.

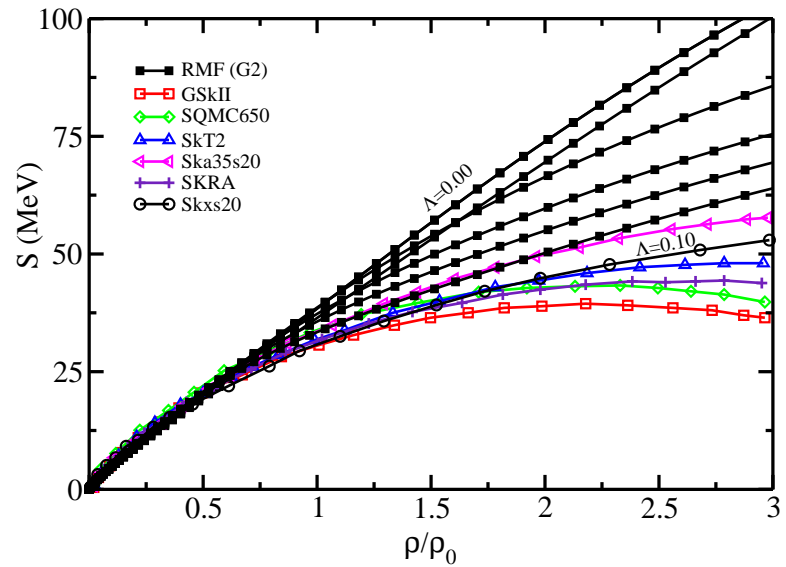

FIG. 2: The results of symmetric energy $E_{s y m}$ is a function of density $\rho$ from RMF for different values of $\Lambda_{v}$ compare with others predictions.

\section{B. Symmetry Energy}

The symmetric energy $E_{\text {sym }}$, which is important in infinite nuclear matter and finite nuclei because of isospin dependent in the interaction. The expression of this key observable is defined as

$$
E_{\text {sym }}(\rho)=\frac{k_{F}^{2}}{6 E_{F}^{*}}+\frac{g_{\rho}^{2} \rho_{B}}{2}\left(\frac{1}{4 m_{\rho}^{* 2}}+\frac{\Lambda_{v} g_{\rho}^{2} g_{\omega}^{2} \omega^{2}}{m_{\rho}^{* 2}}\right),
$$

where the effective energy $\left.E_{F}^{*}=\sqrt{(} k_{F}^{2}+m^{* 2}\right), k_{F}$ is the Fermi momentum and the effective mass $m^{*}=m-g_{S} \phi_{0}$. The effective mass of the $\rho$-meson modified because of nonlinear coupling $(\rho-\omega)$ interaction and is given by

$$
m_{\rho}^{* 2}=\left(1+\eta_{r h o} \frac{g_{\sigma} \sigma}{m_{B}}\right) m_{\rho}^{2}+2 g_{\rho}^{2}\left(\Lambda_{v} g_{v}^{2} \omega_{0}^{2}\right),
$$

where $m_{\rho}$ is the mass of the $\rho$-meson. It is noted that the nonlinear isoscalar-isovector coupling $\left(\Lambda_{v}\right)$ modified the density dependent of $E_{\text {sym }}$ without affecting the saturation properties of the SNM (as shown in Fig. 1 (a)). In this model the symmetric nuclear matter saturates at a Fermi momentum of $k_{F}=$ $1.00 \mathrm{fm}^{1}$ with $E \sim 16.0 \mathrm{MeV}$ and an compressibility of $K_{0}$ $=215 \mathrm{MeV}$ [21]. We can expand the density dependence of the $E_{\text {sym }}$ with the help of these 3-co-efficients as a function of $\mathcal{Y}=\left(\rho-\rho_{0}\right) / 3 \rho_{0}$ as follow:

$$
\begin{aligned}
\mathcal{S}(\rho)= & E_{\text {sym }}\left(\rho_{0}\right)+L_{\text {sym }} \mathcal{Y}+\frac{1}{2} K_{\text {sym }} \mathcal{Y}^{2} \\
& +\frac{1}{6} Q_{\text {sym }} \mathcal{Y}^{3}+O\left(\mathcal{Y}^{4}\right),
\end{aligned}
$$

where $L_{\text {sym }}$ and $K_{\text {sym }}$ are defined as

$$
L_{\text {sym }}=3 \rho_{0}\left(\frac{\partial E_{\text {sym }}}{\partial \rho}\right)_{\rho=\rho_{0}},
$$




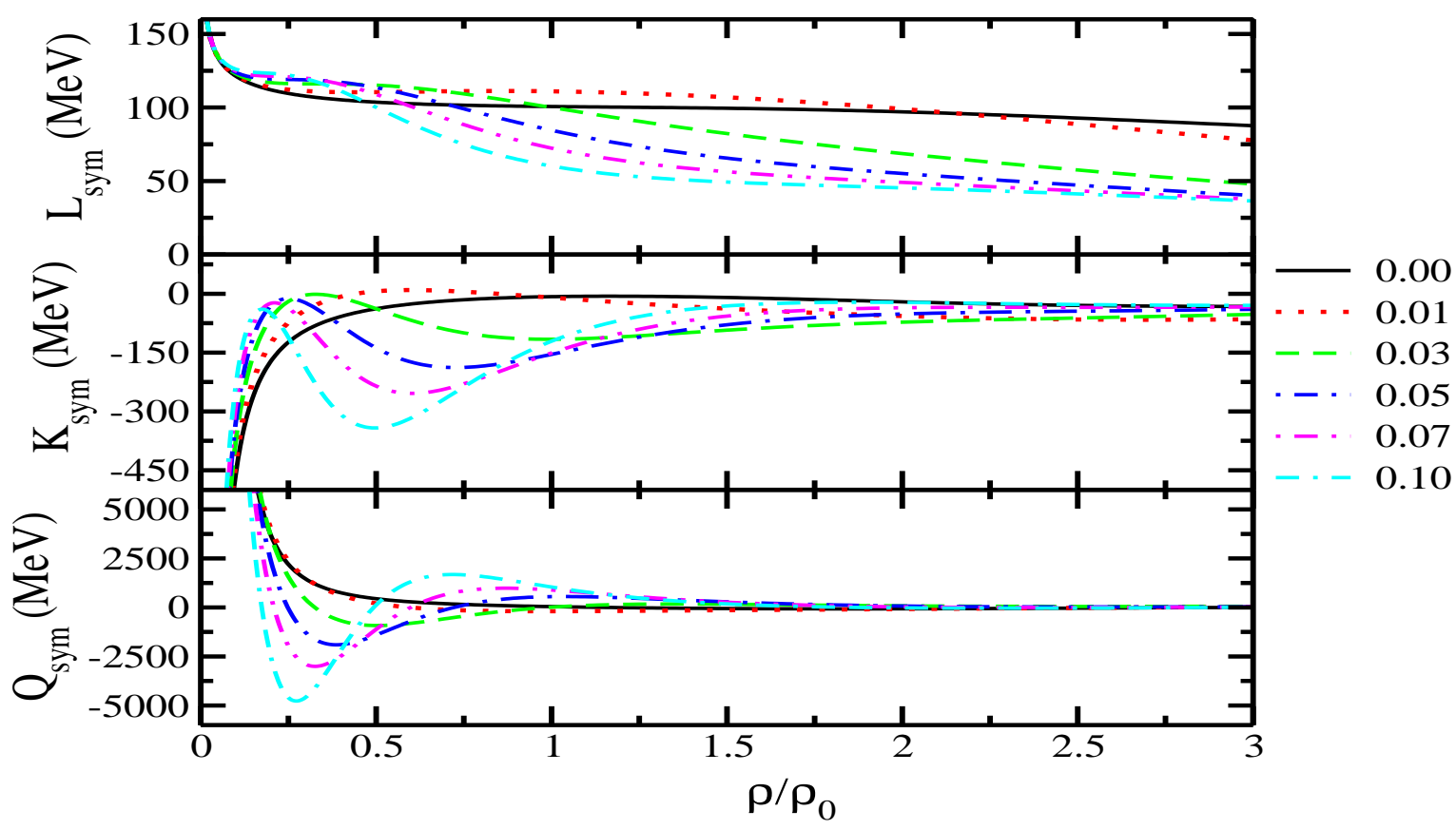

FIG. 3: The slope parameter $L_{s y m}$ along with $K_{s y m}$ and $Q_{s y m}$ for different value of $\Lambda_{v}$, with $G 2$ parameter set.

$$
K_{s y m}=3\left(\rho_{0} \frac{\partial L_{s y m}}{\partial \rho}-L_{s y m}\right)_{\rho=\rho_{0}} .
$$

and

$$
Q_{\text {sym }}=27 \rho_{0}^{3}\left(\frac{\partial^{3} E_{\text {sym }}}{\partial \rho^{3}}\right)_{\rho=\rho_{0}}
$$

Here $L_{s y m}$ is called the slope parameter, which is nothing but the slope of $E_{\text {sym }}$. The quantity $K_{\text {sym }}$ represents as the curvature of $E_{\text {sym }}$ with respect to density. In SNM, the value of $K_{\text {sym }}$ at saturation density $\left(\rho_{0}\right)$ entitled by $K_{0}$ and called as symmetry incompressibility. Finally, $Q_{\text {sym }}$ is the third derivative of the symmetric energy, which called as skewness coefficient [46]. As shown in the Fig. 2, we plotted the $E_{\text {sym }}$ for RMF $\left(\mathrm{G} 2+\Lambda_{v}\right)$ compare with other theoretical predictions [46]. The softness of the $E_{\text {sym }}$ with the coupling $\Lambda_{v}$ clearly observed from the figure. Further, the rate of change of $E_{\text {sym }}$ i.e. the slope parameter $L_{\text {sym }}$ for different values of $\Lambda_{v}$ is displayed in Fig. 3. Both the figure shows similar behaviour with the coupling constant and with baryonic density.

There are many references which trying to fix the constraint on symmetric energy and $L_{\text {sym }}$ [46, 50-55]. Finally we comes to the constraint on $E_{\text {sym }}$ and $L_{s y m}$ at saturation density $\left(\rho_{0}\right)$ and the obtained results are compared with with experimental data at HIC [56], PDR [57, 58], IAS [59] and FRDM [60] in Fig. 4 and Table 2. From the figure, it is clear that for $\Lambda_{v}=0.00$, our observables are not within the experimental constraint region. For the values of $\Lambda_{v}=0.01-0.04$,
TABLE I: The results for slope co-efficient $S_{\text {sym }}, K_{\text {sym }}$ for different value of $\Lambda_{v}$ are in list.

\begin{tabular}{ccc}
\hline$\Lambda_{v}$ & $E_{\text {sym }}$ & $L_{\text {sym }}$ \\
\hline 0.00 & 36.40 & 105.67 \\
0.01 & 38.41 & 111.69 \\
0.02 & 38.87 & 113.09 \\
0.03 & 38.62 & 112.32 \\
0.04 & 38.02 & 110.52 \\
0.05 & 37.27 & 108.27 \\
0.06 & 36.46 & 105.85 \\
0.07 & 35.64 & 103.42 \\
0.08 & 34.86 & 101.05 \\
0.09 & 34.11 & 98.79 \\
0.10 & 33.39 & 96.65 \\
\hline
\end{tabular}

these comes within limit and again go out with further increasing the value of $\Lambda_{v}=0.05$. Hence, from this behavior, we may suggest the limiting value of $\Lambda_{v}$ is $0.01-0.04$, which can improved the observables with $\mathrm{G} 2$ force parameter.

\section{HADRON MATTER}

The density at the interior core of the neutron star is very high $\sim 7-8 \rho_{0}$ so other hadronic part [43, 44, 61-63] also come to play role on the equation of state. In this section, 


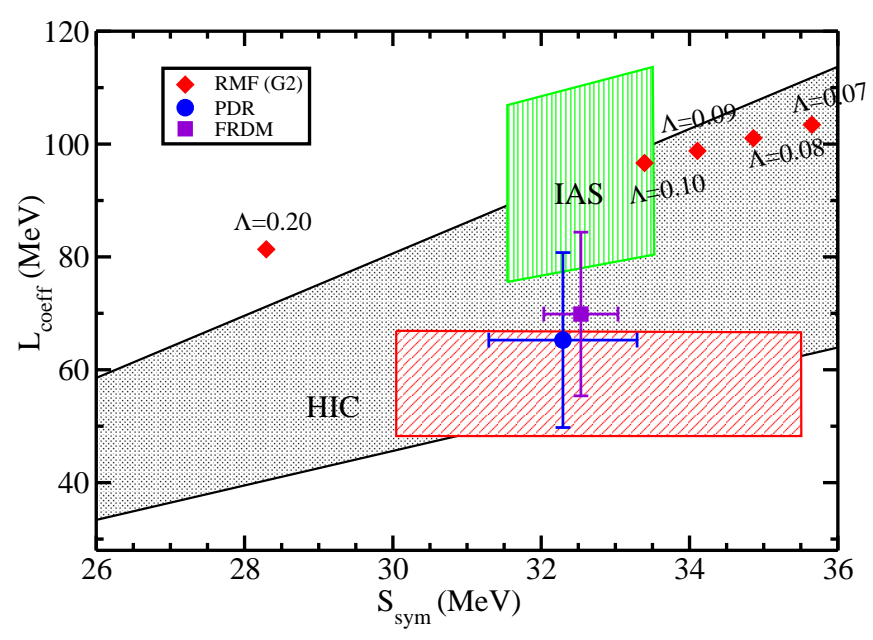

FIG. 4: The Symmetric energy $S_{0}$ with respect to $L_{\text {sym }}$ at saturation density compare with flow data.

we extended our calculations to the system with octet $(n, p$, $\left.\Lambda^{0}, \Sigma^{0, \pm 1}, \Xi^{-, 0}\right)$. The purpose of this work to show the effect of isoscalar-isovector interaction with coupling constant $\Lambda_{v}$ on the equation of state of the nuclear system with full octet. It is worthy to mention that, many authors [21, 41, 64] have been used $G 1$ and $G 2$ forces for the description of finite and infinite nuclear matter properties. Moreover, the non-linear isoscalar-isovector interaction in mean field was discussed in Refs. [65, 66], here we suppose to check this interaction with G2 along with $\Lambda_{v}$, keeping all saturation constraints. We assume that all the hyperon in the octet system have the same coupling ratio with mesons. This is given by a constant factor which is equal to $\sqrt{2 / 3}$ [64], the density of the system $\sim$ 7-8 $\rho_{0}$ and assuming that the stars are the composite system of baryons. The electron $e^{-1}$ and muon $\mu$ are for maintaining the charge neutrality and $\beta$ - equilibrium condition [64, 67] under the week interactions:

$$
B_{1} \rightarrow B_{2}+l+\overline{\nu_{l}} ; \quad B_{2}+l \rightarrow B_{1}+\nu_{l},
$$

where $B_{1}, B_{2}, l, \nu, \bar{\nu}$ are baryons, leptons, neutrino and antinuetrino respectively. In case of octet system, we are dealing with neutron star (real system), which became unstable with small change in the system. The equation of state obtained for nuclear matter with nucleon and hadrons for $G 2+\Lambda_{v}$ is shown in Fig 5.

We plotted pressure $\left(\mathrm{fm}^{-4}\right)$ density with respect to energy density $\left(\mathrm{fm}^{-4}\right)$ in Fig. 5. Here we can see that at high density octet EOS becomes stiffer by increasing the $\Lambda_{v}$ value but it behave different way at low density region. Equation of state for necleon case (EOS-NP) becomes softer with increasing $\Lambda_{v}$ value over whole the density region. We compare our results with the empirical EOS obtained by Steiner et al. [68]. Along with the octet we also plotted nucleon EOS at different $\Lambda_{v}$ values.Nucleon EOS well matches with the data of Steiner et al. [68] at low and high density but the octet EOS is devi- ates at high density. The octet EOS compares well with the

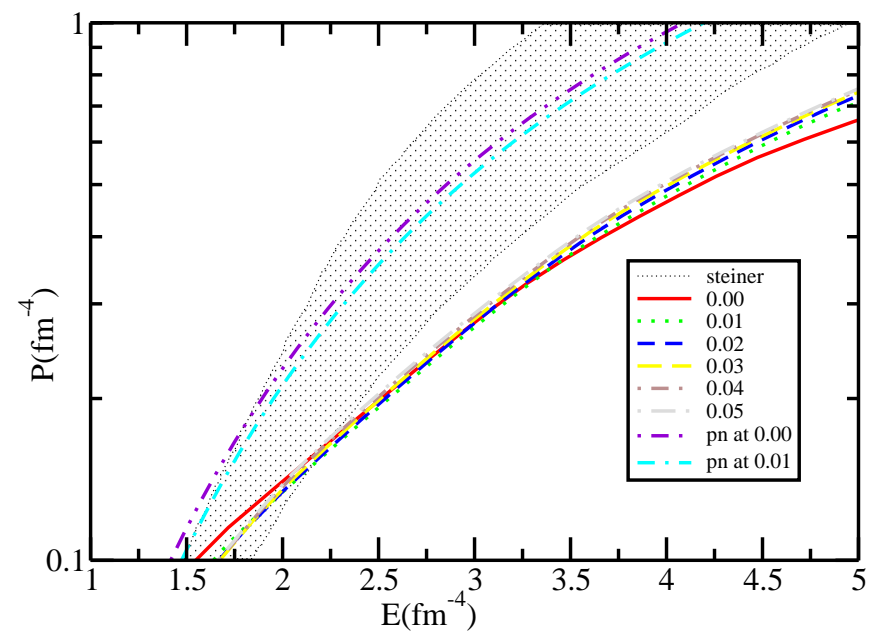

FIG. 5: Equation of states(EOS) with the E-RMF model for octet and $\mathrm{NP}$ at different $\Lambda_{v}$ value.

experimental data only at intermediate density. An impressive observation is noted from this plot, the influence of $\Lambda_{v}$ with G2 parameter set is not much because in G2 already taken the four power of iso-scalar meson so it is more effective then iso-scalar and iso-vector interaction.

\section{SUMMARY AND CONCLUSIONS}

After analyzing the result whatever got from this calculation, we forcefully say that $\Lambda_{v}$ i.e. the coupling of isoscalar and isovector interaction is very crucial to softening the equation of state of nucleonics and octet system. One can easily observed that the $E_{\text {sym }}$ curve with respect to $\rho / \rho_{0}$ became softer with varying $\Lambda_{v}$ but after $\Lambda_{v} \sim 0.05$ it became constant. Same result we got in $L_{\text {sym }}$ which is the slope of symmetric energy. For symmetric nuclear matter isoscalar and isovector interaction do not effect the $E / A$ and pressure density curve with respect to $\rho / \rho_{0}$. We got identical curve as in Ref. [21] for $G 1$ and $G 2$ sets with experimental data [47]. In case of hadron, the extra term containing $\Lambda_{v}$ plays an important role in softening the equation of state. Hence, we may say that this interaction term is crucial for both cases (nucleon and hadron). Further, we extend our calculation to the neutron star properties in near future.

\section{ACKNOWLEDGMENTS}

One of the author MB thanks to Institute of Physics for local hospitality at the time of work. We thanks to B. K. Sharma for important discussions related to this work. 
[1] G. A. Lalazissis, J. Konig, and P. Ring, Phys. Rev. C 55, 540 (1997).

[2] D. Vretenar, A. Wandelt, and P. Ring, Phys. Lett. B 487, 334 (2000).

[3] D. Vretenar, T. Niksc, and P. Ring, Phys. Rev. C 68, 024310 (2003).

[4] G. Colo, P. F. Bortignon, N. Van Gai, A. Bracco, and R. A. Broglia, Phys. Lett. B 276, 279 (1992).

[5] J. P. Blaizot, J. F. Berger, J. Decharge, and M. Girod, Nucl. Phys. A 591, 435 (1995).

[6] I. Hamamoto, H. Sagawa, and X. Z. Zhang, Phys. Rev. C 56, 3121 (1997).

[7] K. A. Brueckner, C. A. Levinson, and H. M. Mahmoud, Phys. Rev. 95, 217 (1954).

[8] H. A. Bethe, Phys. Rev. 103, 1353 (1956).

[9] J. Goldstone, Proc. R. Soc. London, Ser. A 239, 267 (1957).

[10] H. A. Bethe, Annu. Rev. Nucl. Sci. 21, 93 (1971).

[11] M. R. Anastasio, L. S. Celenza, W. S. Pong, and C. M. Shakin, Phys. Rep. 100, 327 (1983).

[12] C. J. Horowitz and B. D. Serot, Phys. Lett. B 137, 287 (1984).

[13] C. J. Horowitz and B. D. Serot, Nucl. Phys. A 464, 613 (1987).

[14] R. Brockmann and R. Machleidt, Phys. Rev. C 42, 1965 (1990).

[15] R. Brockmann and R. Machleidt, Phys. Lett. B 149, 28 (1984).

[16] J. Piekarewicz, Phys. Rev. C 66, 034305 (2002).

[17] J. Piekarewicz, Phys. Rev. C 69, 041301 (2004).

[18] B. K. Agrawal, S. Shlomo, and V. Kim Au, Phys. Rev. C 68, 031304 (2003).

[19] N. K. Glendenning, F. Weber, and S. A. Moszkowski, Phys. Rev. C 45, 844 (1992).

[20] H. Heiselberg and M. Hjorth-Jensen, Astrophys. J. Lett. 525, L45 (1999).

[21] P. Arumugam, B. K. Sharma, P. K. Sahu, S. K. Patra, T. Sil, M. Centelles, and X. Vinas, Phys. Lett. B 601, 51 (2004).

[22] P. K. Panda, A. Mishra, J. M. Eisenberg, and W. Greiner, Phys. Rev. C 56, 3134 (1997).

[23] J. Casares, P. A. Charles, and E. Kuulkers, Astrophys. J. Lett. 493, L39 (1998).

[24] S. Weinberg, Physica A 96, 327 (1979).

[25] J. D. Walecka, Ann. Phys. (NY) 83, 491 (1974).

[26] B. D. Serot and J. D. Walecka, in Advances in Nuclear Physics, edited by J. W. Negele and Erich Vogt (Plenum Press, New York, 1986), Vol. 16, p. 1.

[27] P.-G. Reinhard, Rep. Prog. Phys. 52, 439 (1989).

[28] B. D. Serot, Rep. Prog. Phys. 55, 1855 (1992).

[29] B. D. Serot and J. D. Walecka, Int. J. Mod. Phys. E 6, 515 (1997).

[30] S. L. Shapiro and S. A. Teukolsky, Black Holes, White Dwarfs, and Neutron Stars, Wiley, New York, (1983).

[31] C. J. Horowitz and B. D. Serot, Nucl. Phys. A 368, 503 (1981).

[32] J. Boguta and A. R. Bodmer, Nucl. Phys. A 292, 413 (1977).

[33] Y. K. Gambhir, P. Ring, and A. Thimet, Ann. Phys. (NY) 198, 132 (1990).

[34] P. Ring, Prog. Part. Nucl. Phys. 37, 193 (1996).

[35] R. J. Furnstahl, B. D. Serot, and H. B. Tang, Nucl. Phys. A 598, 539 (1996).

[36] R. J. Furnstahl, B. D. Serot, and H. B. Tang, Nucl. Phys. A 615,
441 (1997).

[37] H. Mu" ller and B. D. Serot, Nucl. Phys. A 606, 508 (1996).

[38] H. Georgi and A. Manohar, Nucl. Phys. B 234, 189 (1984).

[39] H. Georgi, Phys. Lett. B 298, 187 (1993).

[40] J. J. Rusnak and R. J. Furnstahl, Nucl. Phys. A 627, 495 (1997).

[41] R. J. Furnstahl and B. D. Serot, Nucl. Phys. A 671, 447 (2000). $\mathrm{G}$

[42] B. D. Serot, in Lecture Notes in Physics, edited by Georgios A. Lalazissis, Peter Ring, and Dario Vretenar (Springer-Verlag, Berlin, 2004), Vol. 641, p. 31.

[43] I. Bednarek and R. Manka, Int. J. Mod. Phys. D 10, 607 (2001).

[44] I. Bednarek and R. Manka, Phys. Rev. C 73, 045804 (2006).

[45] R. Brockmann, R. Machleidt, Phys. Rev. C 42, 1965 (1990); G.Q. Li, R. Machleidt, R. Brockmann, Phys. Rev. C 45, 2782 (1992).

[46] M. Dutra et al., Phy. Rev. C 85, 035201 (2012).

[47] P. Danielewicz, R. Lacey, W.G. Lynch, Science 298, 1592 (2002).

[48] F. Sammarruca, Int. J. Mod. Phys. E, 19, 1259 (2010).

[49] S. Typel, Phys. Rev. C 71, 064301 (2005).

[50] C. Xu, B.-A. Li, and L.-W. Chen, Phys. Rev. C 82, 054607 (2010).

[51] W. G. Newton, M. Gearheart, and B.-A. Li, arXiv:1110.4043 (2011).

[52] A. W. Steiner and S. Gandolfi, Phys. Rev. Lett. 108, 081102 (2012).

[53] J. M. Lattimer and Y. Lim, arXiv:1110.4043 (2012).

[54] M. Tsang et al., Phys. Rev. C 86, 015803 (2012).

[55] F. J. Fattoyev, W. G. Newton, J. Xu, and B.-A. Li, Phys. Rev. C 86, 025804 (2012).

[56] M. B. Tsang, Y. Zhang, P. Danielewicz, M. Famiano, Z. Li, W. G. Lynch, and A. W. Steiner, Phys. Rev. Lett. 102, 122701 (2009).

[57] A. Klimkiewicz et al., Phys. Rev. C 76, 051603 (2007).

[58] A. Carbone, G. Col 'o, A. Bracco, L.-G. Cao, P. F. Bortignon, F. Camera, and O. Wieland, Phys. Rev. C 81, 041301 (2010).

[59] P. Danelewicz and J. Lee, arXiv:1111.0326v1.

[60] P. Moller, W. D. Meyers, H. Sagawa, and S. Yoshida, Phys. Rev. Lett. 108, 052501 (2012).

[61] N. K. Glendenning, Compact Stars, Nuclear Physics, Particle Physics and General Relativity (Springer-Verlag, New York,(1997).

[62] N. K. Glendenning, Astrophys. J. 293, 470 (1985).

[63] J. Schaffner-Bielich and A. Gal, Phys. Rev. C 62, 034311 (1999).

[64] B. K. Sharma, P. K. Panda, and S. K. Patra, Phy. Rev. C 75, 035808 (2007).

[65] B. G. Todd-Rutel and J. Piekarewicz,Phys. Rev. Lett.95, 122501 (2005).

[66] F. J. Fattoyev, C. J. Horowitz, J. Piekarewicz, and G. Shen Phy. Rev. C 82, 055803 (2010).

[67] A. W. Steiner, S. Reddy, and M. Prakash, Phys. Rev. D 66, 094007 (2002bite

[68] A. W. Steiner, J. M. Lattimer, and E. F. Brown, Astrophys. J. 722, 33 (2010). 